\title{
Global Surgery: The Perspective of Public Health Students
}

\author{
Brittany A. Hout ${ }^{1}$, Eric P. Matthews ${ }^{1} \&$ Jan-Michael Van Gent ${ }^{1}$ \\ ${ }^{1}$ College of Graduate Health Studies, A.T. Still University, Arizona, United States \\ Correspondence: Brittany A. Hout, College of Graduate Health Studies, A.T. Still University, Arizona, United \\ States. Tel: 1-336-460-9266. E-mail: Bahout@atsu.edu
}

Received: April 16, 2020 Accepted: May 10, 2020 Online Published: May 21, 2020

doi:10.5539/gjhs.v12n8p1 URL: https://doi.org/10.5539/gjhs.v12n8p1

\begin{abstract}
Current research has emphasized the importance of increased involvement of medical professionals and global health specialists for the success of global surgery efforts. This quantitative descriptive study aimed to examine public health students' perceptions of global surgery. A 21- question mixed method online survey was distributed over eight weeks via student email to all students enrolled in the Masters of Public Health Program at A.T. Still University (ATSU) College of Graduate Health Studies. Of 212 students, 35 (16.5\%) respondents completed the survey with 30 students reporting interest in global health in their future public health careers. Two-thirds of students erroneously identified infectious diseases as the leading cause of death worldwide, not traumatic injury. Participants identified infectious disease and $\mathrm{OB} / \mathrm{GYN}$ as the two medical fields to contribute significantly to global health. Surgical care was felt to be the least economically cost-effective medical field for low and middle-income countries (LMICs). As the first project to report perspectives of public health students regarding global surgery, this study highlighted several significant misconceptions concerning global surgery. Like the results from similar studies in medical students, it is alarming that there is such a paucity of community health knowledge surrounding surgery and its effects on global surgical needs. Further research should focus on the effect on student perceptions after curriculum modification include education regarding the burden of surgical disease and role of global surgery.
\end{abstract}

Keywords: global surgery, global health, public health students, curriculum

\section{Introduction}

In the last two decades, global health has become an essential topic. The right to health care has polarized global health into key social, economic, and political interests (Dare et al., 2014). Historically, the focus of global health has been dominated by infectious diseases such as HIV/AIDS, tuberculosis, and malaria (Kennedy, Fairfield, \& Fergusson, 2015). Industrialization, developed health care systems, and successful global health programs have created a shift in global health priorities from infectious disease to non-communicable and surgically treatable pathology (Kennedy et al., 2015). While remarkable efforts have improved the multifaceted burden of disease (infectious, maternal, and noncommunicable disease) in low and middle-income countries (LMICs), surgical care has not been included in any these global health initiatives, until recently. The growing burden of surgical disease and the need for equitable access to surgical care in LMICs has increased interest in global surgery within the global health community. This study aimed to understand and characterize public health students' perceptions of global surgery.

\subsection{Background}

Generalized awareness for the need of surgical intervention increased after Farmer and Kim (2008) first broached the topic of global surgery with their work that highlighted the overwhelming burden of surgical disease and disparity in care for LMICs. Unfortunately, this burden of disease is heaviest in rural LMICs where there is a significant disparity in care (Ologunde, Maruthappu, Shanmugarajah, \& Shalhoub, 2014). In 2010, 17 million preventable deaths ( $32.9 \%$ of all deaths worldwide) were attributed to surgically treatable conditions (Meara et al., 2015). The vast majority of these conditions include surgical trauma related to motor vehicle and farming accidents, acute surgical abdominal processes, obstetric and gynecologic emergencies such as post-partum hemorrhage, and congenital birth defects. With the shift in epidemiology and failure to include global surgery in the global health platform, global health experts report that surgical disease now accounts for more deaths annually than HIV, malaria, and tuberculosis combined (Kant, Roy, \& Zodpey, 2018). Just as many individuals are left 
chronically disabled and/or with shorter lifespans due to conditions requiring surgical interventions than due to the aforementioned infectious diseases.

\subsection{Current Iniatives in Global Surgery}

Historically, diverse groups including nongovernmental organizations (NGOs), academic institutions, and missionary organizations have tried to combat the burden of surgical disease by providing direct care or surgical education (Shrime, Sleemi, \& Ravilla, 2014). A majority of existing initiatives for global surgery in LMICs are predominantly provided on a charitable platform by these key stakeholders. Currently, there is not a standard model of care or education used for global surgery (Tollefson, \& Larrabee, 2012). The lack of established evidence-based interventions, likely due to the vast resource difference, has created significant fragmentation among specialists, and has been named as a leading hindrance for the global surgery initiative (Shiffman, 2017).

\subsection{Rationale}

Current research has emphasized the importance of increased involvement of medical professionals and global health specialists for global surgery efforts (Meara et al., 2015). Students have been considered as a critical element for the long-term success of global health efforts because they are the driving force to engage, develop, and implement policy and programs. Existing literature examines the consequences of medical students' perceptions of global surgery (Mehta et al., 2017). The researchers highlighted the cascading effect of how perceptions can influence clinical experiences, research opportunities, and career tracks (Mehta et al., 2017). Medical students have primarily been the focus on evaluation for this topic because surgeons and anesthesiologists have been the primary champions for the global surgery community (Shiffman, 2017). Due to the lack of involvement of public health officials in this initiative, there has been a prominent gap in research assessing the population of public health students. The purpose of this study is to close this gap by examining public health students' perceptions of global surgery.

\section{Method}

\subsection{Research Survey}

The research tool used in this study was a survey questionnaire. A 21-question electronic survey was created by modifying an existing survey (Mehta et al., 2017). Permission to use and modify the research survey was requested and granted by the original survey authors. Several questions were adjusted to account for the difference in research populations. The mixed method questionnaire consisted of multiple-choice questions, free text, and the five-point Likert scale to characterize public students' perceptions of the use of global surgery. The survey was divided into four parts measuring student demographics, perceptions of the role of surgery in global health, the impact of global surgery and perceived barriers for global surgery on the global health agenda. Although the original research tool was validated in 2017 , the modified survey was reviewed by subject matter experts with consideration of a different research population prior to its use in this study.

\subsection{Study Population and Survey Implementation}

The study participants for this quantitative descriptive study were students currently enrolled in the A.T. Still University (ASTU) Masters of Public Health (MPH) Program. The online program, managed through the College of Graduate Health Studies, had 212 students who were eligible to be included in the study. Some students were enrolled as part of a dual-degree program which allowed them to graduate with a medical degree and MPH. Faculty and staff were excluded from participation. There were no age, gender, or experience exclusion standards for this study.

After obtaining project approval from the ATSU Institutional Review Board and College of Graduate Health Studies, study participants were contacted in July 2019 through their official ATSU university email for the opportunity to participate in the survey. The email contained a short summary introduction regarding participation information and a link to the electronic survey. The survey was conducted through Survey Monkey, a platform utilized for the creation and distribution of cost-free, anonymous, online surveys. Students were given eight weeks to complete the survey. Three reminder emails were sent to the study population throughout the data collection period.

\subsection{Data Analysis}

All completed survey questionnaire data was transferred from Survey Monkey into SPSS database. Results were analyzed by student year since the elective global health course occurs during the second year at A.T Still University. Due to the difference in medical school curriculum and experience, the results were also analyzed based on participants who are currently pursuing and / or have completed a medical degree. Survey responses with 
written answers were evaluated and recoded, if needed, for data analysis. Several survey questions were answered with more than the required responses. In this case, the first two selected responses were used for data analysis. Once all survey results had been collected, cleaned, and recoded as needed, the survey data was manually entered and validated using IBM SPSS Advanced Statistics, version 26.0 (5725-A54). Data analysis was performed with SPSS statistical software for tabulation and chi-square tests ( $p$-value $<0.05$ represented statistical significance).

\section{Results}

Of 212 available participants, $36(17 \%)$ students completed the research survey. One completed survey was excluded from the results because the participant answered "no" on the first question of the survey granting participant consent. The final number of participants included for analysis was 35 . Of the 35 consenting participants, seven $(20.0 \%)$ identified themselves as first year public health students, $22(62.9 \%)$ identified as second year public health students, and six (17.1\%) reported as other status (Table 1). The other category was further self-expanded as third year dual MPH / DO program or recently graduated by participants. Two of the six students who reported as currently enrolled in the program as other status were recently graduated from the MPH program. These students were not excluded from the study since their graduation dates were unknown and they were still engaged in the university email system. Eight students total $(22.9 \%$ total: $18.2 \% \mathrm{MPH}$ second year, $66.7 \%$ Other) are currently pursuing or have completed MD/DO training. The majority of participants were females (77.1\%) aged 25-34 years old (71.4\%). Twenty-nine participants (82.9\%) were born in the United States and have never lived outside of the country for more than one year. A quarter of the students in this study reported having experience with a foreign medical system. Six of the nine respondents $(66.7 \%)$ with foreign medical experience were born or raised outside of the United States.

Professional aspirations were queried of every participant. Only 10 of the 35 (28.6\%) students were planning to pursue a career in academics or teaching. Two (20\%) of these students were enrolled or have completed a medical degree program. Of the 35 respondents, 30 students (85.7\%) envisioned incorporating global health work into their public health careers. There was an increase in percentage of students interested in global health work between the first $(5 / 7 ; 71.4 \%)$ and second year $(19 / 22 ; 86.4 \%)$ of the ATSU MPH program. Of note, all six students that identified as other status reported envisioning a global health career. Thirty students have taken or plan to take a course in global health issues ( $85.7 \%$ total: $20.0 \%$ first year; $54.3 \%$ second year; $11.4 \%$ Other; 0.229$)$. Seven of eight $(87.5 \%)$ students currently enrolled or have completed a medical degree program are planning to incorporate global health work into their careers.

A majority of participants felt the leading cause of annual mortality rates worldwide were related to infectious diseases $(68.6 \%)$ or obstetric complications $(5.7 \%)$. Only $25.7 \%$ of respondents correctly answered traumatic injury was the leading cause of mortality worldwide. There was greater variance in answers in second year students compared to the groups of respondents. For students who have or are currently pursuing a medical degree, there was no statistical significance found in correctly identifying the leading cause of annual mortality rates worldwide $(3 / 8 ; 37.5 \% ; \mathrm{p}=0.546)$.

When asked about their own personal understanding of the term global surgery, 12 students answered they were unfamiliar with the term (34.3\% total: $25.0 \%$ first year, $66.7 \%$ second year, $8.3 \%$ Other, 0.773$)$. The majority of participants $(22 / 35,62.9 \%)$ felt the second definition presented best fit their understanding of global surgery. There were no respondents who answered global surgery represented a global health platform to them. Answers did not significantly differ for students who are currently pursuing or already have medical degree (Graph 2).

Over half of all participants $(22 / 35 ; 62.9 \%)$ felt infectious disease was one of the two medical fields that have had the greatest impact on global health over the last 25 years. Obstetrics and gynecology was ranked second with 18 students $(51.4 \%)$. There was a statistical significance noted $(\mathrm{p}=0.040)$ that only two of the seven $(28.6 \%)$ first year students felt $\mathrm{OB} / \mathrm{GYN}$ had made significant contributions to global health compared to $50.0 \%$ of second year students and $83.3 \%$ of Other ATSU MPH students. When asked which two medical fields will have the greatest impact on global health over the next 25 years, OB/GYN (13/34, 38.2\%) and infectious disease $(11 / 34,32.4 \%)$ were again selected as the first and second choice answers by participants respectively. A quarter of participants felt surgical care had potential for significant future impact on global health $(9 / 35,26.5 \%)$. Of the eight participants who selected surgical care, none of these respondents were first year MPH students. Neurology was chosen as the least represented medical field in the global health agenda $(20 / 35,57.1 \%)$, while Psychiatry was identified as the second least represented medical field $(18 / 34,52.9 \%)$. Only three participants $(3 / 34,8.8 \%)$ felt surgery was under represented in the global health agenda. Family practice was selected by over half of students $(19 / 35 ; 54.3 \%)$ as the medical field that most contributes to a comprehensive healthcare system. Only one student, a second year student, identified surgical care as being the best indicator of an adequate health care system. 
Students compared the capabilities of five medical fields including family practice, OB/GYN, infectious disease, emergency medicine, and surgical care using the Likert scale. Eight participants felt surgical care $(8 / 35 ; 22.9 \%)$ almost never provided preventive medical care while family medicine was seen as the medical field most focused on preventive medical care $(10 / 35 ; 28.6 \%)$. The majority of students $(31 / 35 ; 88.6 \%)$ felt surgery had the potential to provide preventive care sometimes. Surgical care was found to be the least economically cost-effective medical field for resource-poor countries.

External frame, defined as public portrayal and misconceptions of global surgery, was reported as the most common perceived barrier and challenge for the inclusion of global surgery on the global health agenda (48.6\%). The second most common perceived barrier identified by students was global governance (30.3\%).

\subsection{Tables}

Table 1. Demographic Characteristics of Study Sample

\begin{tabular}{|c|c|c|c|c|c|}
\hline Characteristic & All & $\begin{array}{l}\text { MPH } \\
1^{\text {st }} \text { Year }\end{array}$ & $\begin{array}{l}\text { MPH } \\
2^{\text {nd }} \text { Year }\end{array}$ & Other & P Value \\
\hline Participants, n & 35 & 7 & 22 & 6 & \\
\hline Gender & & & & & .040 \\
\hline Female & 27 & 4 & 20 & 3 & \\
\hline Male & 8 & 3 & 2 & 3 & \\
\hline Age, Range in Years & & & & & .020 \\
\hline $18-24$ & 2 & 1 & 0 & 1 & \\
\hline $25-34$ & 25 & 1 & 19 & 5 & \\
\hline $35-44$ & 4 & 2 & 2 & 0 & \\
\hline $45-54$ & 3 & 2 & 1 & 0 & \\
\hline $55-64$ & 0 & 0 & 0 & 0 & \\
\hline $65-74$ & 1 & 1 & 0 & 0 & \\
\hline 75 and older & 0 & 0 & 0 & 0 & \\
\hline Place of birth & & & & & .386 \\
\hline United States & 29 & 5 & 18 & 6 & \\
\hline International & 6 & 2 & 4 & 0 & \\
\hline Lived abroad for $>1$ year & & & & & .386 \\
\hline Yes & 6 & 5 & 4 & 0 & \\
\hline No & 29 & 2 & 18 & 6 & \\
\hline Foreign Medical System Experience & & & & & .281 \\
\hline Yes & 9 & 2 & 4 & 3 & \\
\hline No & 26 & 5 & 18 & 3 & \\
\hline
\end{tabular}

Note: ${ }^{*} p<.05$ 


\subsection{Figures and Sketches}

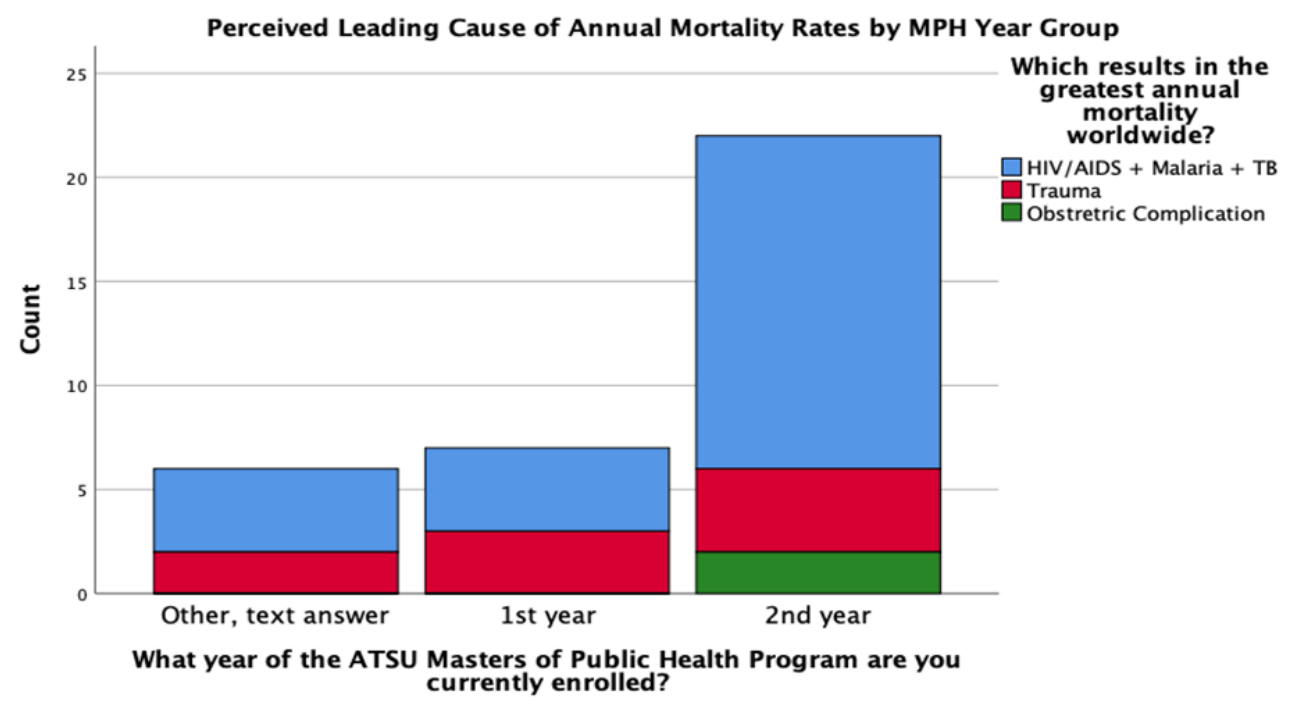

Graph 1. Perceived Leading Cause of Annual Mortality Rates by MPH Year Group

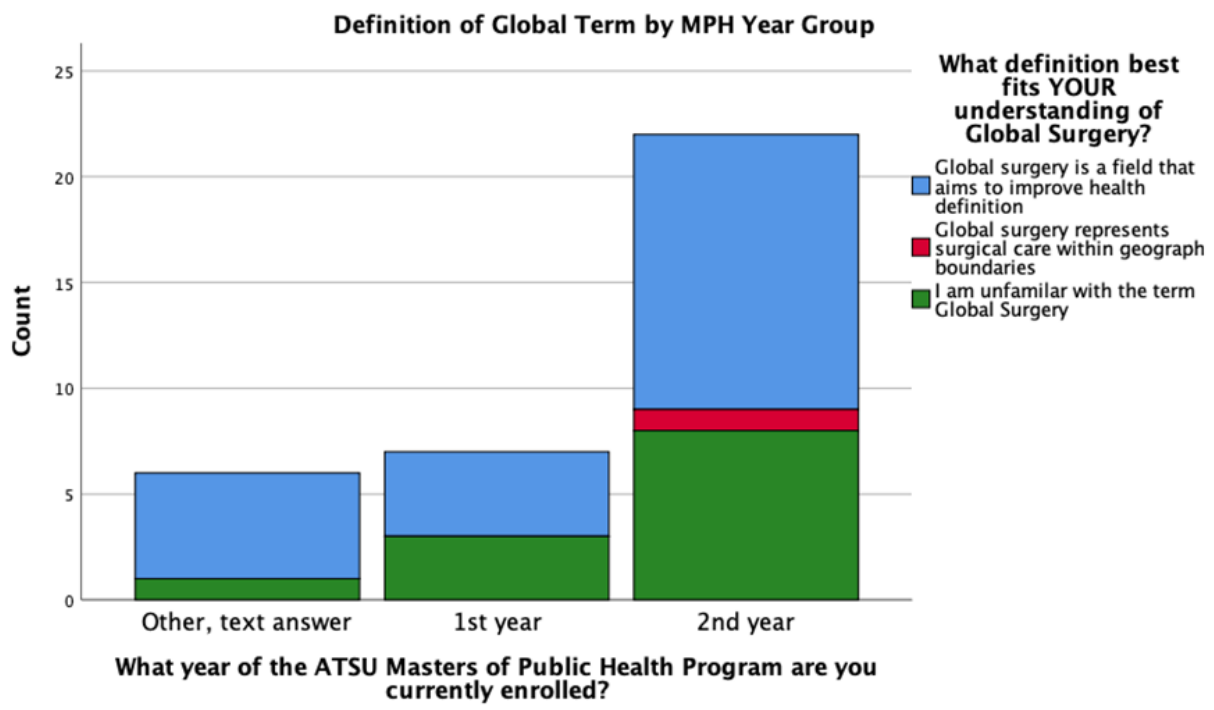

Graph 2. Definition of Global Surgery by MPH Year Group

\section{Discussion}

To further highlight the importance of academic partnerships in global surgery and address a significant gap in research, this study examined public health students' perceptions of global surgery. We found that public health students were not aware of the unmet global burden of surgical disease and did not recognize the importance of surgical care in global health. When asked about the leading cause of annual mortality rates worldwide, the majority of participants $(68.6 \%)$ misidentified infectious diseases as the etiology instead of traumatic injury. Additionally, public health students identified infectious disease and $\mathrm{OB} / \mathrm{GYN}$ as the two medical fields that have had, and will have the greatest effect on global health. Only one student felt surgical care was an indicator of an adequate healthcare system. The lack of awareness regarding the global burden of surgical disease and consequence of inadequate access to quality surgical care in LMICs contribute to the underappreciation of surgical care in global health from public health students. The results from this study also demonstrated public health students do not view global surgery as feasible or cost-effective.

These perceptions may be an unintentional byproduct from the successful, well-known and established global 
health platforms aimed to combat infectious disease and improve women's health (Kennedy et al., 2015). Moreover, public health student perceptions are likely influenced by the agenda from a community health centered curriculum. Traditionally, the MPH curriculum has been centered around five core disciplines of public health: biostatistics, environmental health science, epidemiology, health policy and management, and social and behavioral sciences (Winskell, Evans, Stephenson, Del Rio, \& Curran, 2014). The increase in globalization and subsequent influence to population health has necessitated training of public health professionals in global health perspectives. As a dynamic and upcoming field, global health creates unique challenges in curriculum development for MPH programs. Without curriculum standardization, the majority of MPH programs offer elective global health tracks that emphasize the key global health thematic areas while surgical care is often and unfortunately absent (Winskell et al., 2014). The magnitude of the lack of exposure to global surgery in the MPH curriculum was best appreciated when participants (35\%) were unable to give their personal understanding of the term global surgery because they were unfamiliar with the concept.

\subsection{Comparison to Similar Studies}

When survey results were compared to a similar study completed using medical students enrolled at Johns Hopkins Medical School, it was noted the students shared several perceptions (Mehta et al., 2017). Results demonstrated the majority of public health students and medical students incorrectly identified infectious disease as the leading cause of annual worldwide mortality rates (Mehta et al., 2017). Students felt primary care best represented a complete health system. Additionally, surgical care was ranked last by both public health and medical students as having the potential to practice preventive medicine and the feasibility to be practiced cost-effectively in a resource limited setting (Mehta et al., 2017).

While there were some similarities between the two sets of students, several differences were also observed. Over twice as many public health students (85.7\%) envisioned incorporating global health work into their careers compared to medical students (41\%). Medical students identified surgical care as the second ranked medical field to have the greatest influence in global health in the next 25 years (Mehta et al., 2017). Twenty percent of medical students felt surgical care was one of the two fields that have had the greatest influence in global health in the last 25 years (Mehta et al., 2017). In contrast, surgical care was not selected as a major contributing medical field to the past or present of global health by public health students.

\subsection{Limitations to Research}

This study has several limitations. First, the sample methodology and size were restricted by geographical, financial, and time constraints. The survey responses were from a single university which may not be representative of public health students' perceptions at other institutions. Due to the geographical location of the researcher during the data collection phase, the survey was distributed electronically for convenience. Furthermore, the sample size was small with only $35(16.5 \%)$ of 212 students participating in the study. It is recognized that a small sample size creates a large standard of error. Despite the sample size not meeting the goal of at least 100 participants, the response rate was greater the average expectant response rate of $10-15 \%$ for external surveys. Three reminder emails were sent to students encouraging participation, however, the response rate was likely affected by the lack of participation incentive and perceived benefit for contribution. Incentives for participation were not offered during this study because funding was not available. As the first project to report perspectives of MPH students towards global surgery, the noted limitations open up future research opportunities in this population.

\subsection{Recommedations and Practical Application}

Public health students' interest in global health can be noted in this study and from the incorporation of global health competencies into the public health curriculum. This study highlights several significant misconceptions of global health that negatively impact public the perception of global surgery. Public health programs should continue to development global health curriculums to reflect principal issues and advances in the field. Specifically, attention should be placed on updating core global health education competencies regarding the epidemiologic shift from infectious disease to trauma in an effort to better understand the global burden of surgical disease. Public health student misperceptions may be corrected by integrating learning objectives concerning global surgery. Additionally, global health courses should be taught by faculty who are proficient in global health studies. Institutions should promote global health opportunities for faculty trained in traditional public health disciplines that lack global health perspective, expertise and experience.

\section{Conclusion}

When queried about their perceptions of global surgery, public health students were ignorant on the global burden 
of surgical disease, did not feel surgical care had or will have a significant use in global health, and did not view global surgery as feasible or cost-effective. Compared to findings in a similar study done with medical students at Johns Hopkins, this study demonstrated public health students have several noteworthy misconceptions of global surgery that are unique to their population. Further research is warranted to investigate the result and effect of public students' perceptions of global surgery from public health curriculum adjustments to enhance global health studies.

\section{Acknowledgments}

The authors received no financial support for the research, authorship, and/or publication of this article. There are no conflicts of interest to disclose.

\section{Competing Interests Statement}

The authors declare that there are no competing or potential conflicts of interest.

\section{References}

Dare, A. J., Grimes, C. E., Gillies, R., Greenberg, S. L. M., Hagander, L., Meara, J. G., \& Leather, A. J. M. (2014). Global surgery: Defining an emerging global health field. Lancet (London, England), 384(9961), 2245-2247. https://doi.org/10.1016/S0140-6736(14)60237-3

Farmer, P. E., \& Kim, J. Y. (2008). Surgery and global health: A view from beyond the OR. World Journal of Surgery, 32(4), 533-536. https://doi.org/10.1007/s00268-008-9525-9

Kant, L., Roy, N., \& Zodpey, S. P. (2018). Surgical conditions-A neglected aspect of public health: Call to action. Indian Journal of Public Health, 62(3), 211. https://doi.org/10.4103/ijph.IJPH_3_18

Kennedy, E. D., Fairfield, C. J., \& Fergusson, S. J. (2015). A neglected priority? The importance of surgery in tackling global health inequalities. Journal of Global Health, 5(1), 010304. https://doi.org/10.7189/jogh.05.010304

McQueen, K. A. K., Ozgediz, D., Riviello, R., Hsia, R. Y., Jayaraman, S., Sullivan, S. R., \& Meara, J. G. (2010). Essential surgery: Integral to the right to health. Health and Human Rights, 12(1), 137-152.

Meara, J. G., Leather, A. J. M., Hagander, L., Alkire, B. C., Alonso, N., Ameh, E. A., ... Yip, W. (2015). Global surgery 2030: Evidence and solutions for achieving health, welfare, and economic development. The Lancet, 386(9993), 569-624. https://doi.org/10.1016/S0140-6736(15)60160-X

Mehta, A., Xu, T., Murray, M., \& Casey, K. M. (2017). Medical student perceptions of global surgery at an academic institution: Identifying gaps in global health education. Academic Medicine: Journal of the $\begin{array}{lllll}\text { Association of American } & \text { Medical }\end{array}$ https://doi.org/10.1097/ACM.0000000000001832

Ologunde, R., Maruthappu, M., Shanmugarajah, K., \& Shalhoub, J. (2014). Surgical care in low and middle-income countries: Burden and barriers. International Journal of Surgery, 12(8), 858-863. https://doi.org/10.1016/j.ijsu.2014.07.009

Roa, L., Jumbam, D. T., Makasa, E., \& Meara, J. G. (2019). Global surgery and the sustainable development goals. British Journal of Surgery, 106(2), e44-e52. https://doi.org/10.1002/bjs.11044

Rosenberg, J. S. (2016). Global surgical ecosystems: A need for systems strengthening. Annals of Global Health, 82(4), 605-613. https://doi.org/10.1016/j.aogh.2016.09.011

Shiffman, J. (2017). Four challenges that global health networks face. International Journal of Health Policy and Management, 6(4), 183-189. https://doi.org/10.15171/ijhpm.2017.14

Shrime, M. G., Sleemi, A., \& Ravilla, T. D. (2014). Charitable platforms in global surgery: A systematic review of their effectiveness, cost-effectiveness, sustainability, and role training. World Journal of Surgery, 39(1), 10-20. https://doi.org/10.1007/s00268-014-2516-0

Tollefson, T. T., \& Larrabee, W. F., Jr. (2012). Global surgical initiatives to reduce the surgical burden of disease. JAMA, 307(7), 667-668. https://doi.org/10.1001/jama.2012.158

Winskell, K., Evans, D., Stephenson, R., Del Rio, C., \& Curran, J. W. (2014). On academics incorporating global health competencies into the public health curriculum. Public Health Reports, 129(2), 203-208. https://doi.org/10.1177/003335491412900216 


\section{Copyrights}

Copyright for this article is retained by the author(s), with first publication rights granted to the journal.

This is an open-access article distributed under the terms and conditions of the Creative Commons Attribution license (http://creativecommons.org/licenses/by/4.0/). 